\title{
Sensitivity of non-autonomous discrete dynamical systems revisited
}

\author{
Xian-Feng Ding ${ }^{\mathrm{a}}$, Tian-Xiu Lu, ${ }^{\mathrm{b}, *}$, Jian-Jun Wang ${ }^{\mathrm{c}}$ \\ a School of Sciences, Southwest Petroleum University, Chengdu, Sichuan, 610500, People's Republic of China. \\ ${ }^{b}$ School of Mathematics and Statistics, Sichuan University of Science and Engineering, Zigong, Sichuan, 643000, People's Republic of \\ China. \\ ${ }^{c}$ Department of Applied Mathematics, Sichuan Agricultural University, Ya'an, Sichuan, 625014, People's Republic of China.
}

Communicated by Y.-B. Xiao

\begin{abstract}
In this note, we construct a transitive non-autonomous discrete system with strongly periodic density which is not sensitive. Besides, we prove that every transitive non-autonomous discrete system with almost periodic density is syndetically sensitive, provided that it converges uniformly to a map, and that a product system is multi-sensitive (resp., $\mathscr{F}$-sensitive) if and only if there exists a factor system is multi-sensitive (resp., $\mathscr{F}$-sensitive), where $\mathscr{F}$ is a filterdual. (C)2017 All rights reserved.
\end{abstract}

Keywords: Non-autonomous discrete system (NADS), sensitivity, $\mathscr{F}$-sensitivity, transitivity, product system.

2010 MSC: 37B55, 54H20.

\section{Introduction}

Let $\left\{f_{\mathfrak{n}}\right\}_{\mathfrak{n}=1}^{\infty}$ be a sequence of continuous self-maps on a compact metric space $(X, \rho)$ and $\mathbb{N}=\{1,2,3, \cdots\}$, $\mathbb{Z}^{+}=\{0,1,2, \cdots\}$. For any positive integers $i$ and $n$, we set $F_{i}^{n}=f_{i+n-1} \circ \cdots \circ f_{i}$ and $f_{i}^{0}=i d_{X}$ and call $\left(X,\left\{f_{n}\right\}_{n=1}^{\infty}\right)$ a non-autonomous discrete system (NADS), where idX is the identity map on $X$. The orbit of any point $x \in X$ is the set $\operatorname{orb}\left(x,\left\{f_{n}\right\}_{n=1}^{\infty}\right):=\left\{F_{1}^{n}(x) \mid n \in \mathbb{Z}^{+}\right\}$. In other words, the solution of the non-autonomous difference equation

$$
\left\{\begin{array}{l}
x_{n+1}=f_{n+1}\left(x_{n}\right), \\
x_{0}=x
\end{array}\right.
$$

Non-autonomous discrete systems were introduced in [7] (see also [6]) and, as we can see, they also appear connected to some non-autonomous difference equations (see $[3,4])$. Note that if $f_{n}=f$ for any $n \in \mathbb{N}$, then the pair $(X, f)$ is a 'classical' autonomous dynamical system (ADS).

For $U, V \subset X$, define the return time set from $U$ to $V$ as $N(U, V)=\left\{n \in \mathbb{Z}^{+} \mid F_{1}^{n}(U) \cap V \neq \varnothing\right\}$.

According to Lan [8], a point $x \in X$ is periodic, if $F_{1}^{n}(x)=x$ for some $n \in \mathbb{N}$ and it is strongly periodic, if there exists $n \in \mathbb{N}$ such that for any $j \in \mathbb{N}, F_{1}^{j n}(x)=x$. The set of all periodic points and all strongly periodic points of $\left\{f_{n}\right\}_{n=1}^{\infty}$ are denoted by $\operatorname{Per}\left(\left\{f_{n}\right\}_{n=1}^{\infty}\right)$ and $\operatorname{SP}\left(\left\{f_{n}\right\}_{n=1}^{\infty}\right)$, respectively.

\footnotetext{
${ }^{*}$ Corresponding author

Email addresses: dingxianfengswpu@163.com (Xian-Feng Ding), lubeeltx@163.com (Tian-Xiu Lu), jianjunw55@163.com (Jian-Jun Wang)
}

doi:10.22436/jnsa.010.10.10 
$\operatorname{A} \operatorname{NADS}\left(X,\left\{f_{\mathfrak{n}}\right\}_{\mathfrak{n}=1}^{\infty}\right)$ is:

(1) (topologically) transitive, if for any pair of nonempty open subsets $U, V$ of $X, N(U, V) \neq \varnothing$;

(2) sensitively dependent on initial conditions (briefly, sensitive), if there exists $\varepsilon>0$ such that for any $x \in X$ and any neighborhood $U$ of $x$, there exist $y \in U$ and $n \in \mathbb{Z}^{+}$satisfying $\rho\left(F_{1}^{n}(x), F_{1}^{n}(y)\right)>\varepsilon$;

(3) equicontinuous, if for any $\varepsilon>0$, there exists $\delta>0$ such that for any $x, y \in X$ with $\rho(x, y)<\delta$ and any $n \in \mathbb{Z}^{+}, \rho\left(F_{1}^{n}(x), F_{1}^{n}(y)\right)<\varepsilon$.

Recall some basic concepts related to the Furstenberg families (see [1] for more details). Let $\mathcal{P}$ be the collection of all subsets of $\mathbb{Z}^{+}$. We say that a collection $\mathscr{F} \subset \mathcal{P}$ is a Furstenberg family, if it is hereditary upwards, i.e., $F_{1} \subset F_{2}$ and $F_{1} \in \mathscr{F}$ imply $F_{2} \in \mathscr{F}$, and is proper, if it is a proper subset of $\mathcal{P}$, i.e., neither empty nor the whole $\mathcal{P}$. In this paper all Furstenberg families considered are proper. It is not hard to see that a family $\mathscr{F}$ is proper, if and only if $\mathbb{Z}^{+} \in \mathscr{F}$ and $\varnothing \notin \mathscr{F}$. Given a family $\mathscr{F}$, we define its dual family as

$$
\kappa \mathscr{F}=\left\{\mathrm{F} \in \mathcal{P} \mid \mathbb{Z}^{+} \backslash \mathrm{F} \notin \mathscr{F}\right\} .
$$

It is easy to check that $\kappa \mathscr{F}$ is a Furstenberg family, and is proper if $\mathscr{F}$ is so. For $i \in \mathbb{Z}^{+}$and $\mathrm{F} \in \mathcal{P}$, let $F+i=\{j+i \mid j \in F\} \cap \mathbb{Z}^{+}$and $F-i=\{j-i \mid j \in F\} \cap \mathbb{Z}^{+}$. A Furstenberg family $\mathscr{F}$ is said to be translation invariant, if for any $\mathrm{F} \in \mathscr{F}$ and any $i \in \mathbb{Z}^{+}, \mathrm{F}+i \in \mathscr{F}$ and $\mathrm{F}-i \in \mathscr{F}$. Given two Furstenberg families $\mathscr{F}_{1}$ and $\mathscr{F}_{2}$, define $\mathscr{F}_{1} \cdot \mathscr{F}_{2}=\left\{\mathrm{F}_{1} \cap \mathrm{F}_{2} \mid \mathrm{F}_{1} \in \mathscr{F}_{1}, \mathrm{~F}_{2} \in \mathscr{F}_{2}\right\}$. A Furstenberg family $\mathscr{F}$ is a filter, if it is proper and satisfies $\mathscr{F} \cdot \mathscr{F} \subset \mathscr{F}$. If $\mathrm{K} \mathscr{F}$ is a filter, then $\mathscr{F}$ is called a filterdual.

Let $\mathscr{F}_{\text {inf }}$ be the collection of all infinite subsets of $\mathbb{Z}^{+}$and $\mathscr{F}_{\text {cf }}$ the family of cofinite subset, i.e., the collection of subsets of $\mathbb{Z}^{+}$with finite complements. It is easy to see that $\mathscr{F}$ inf is the largest proper translation invariant family and its dual $\mathscr{F}_{\mathrm{cf}}=\kappa \mathscr{F}_{\text {inf }}$, clearly as a filter is the smallest one.

A subset $F=\left\{a_{1}<a_{2}<\cdots\right\} \subset \mathbb{Z}^{+}$is:

(1) syndetic, if there exists an $N \in \mathbb{Z}^{+}$such that $a_{i+1}-a_{i} \leqslant N$ for all $i \in \mathbb{N}$;

(2) thick, if for any $i \in \mathbb{N}$ there exists $a_{i} \in \mathbb{Z}^{+}$such that $\left\{a_{i}, a_{i}+1, \cdots, a_{i}+i\right\} \subset F$;

(3) thickly syndetic, if for any $k \in \mathbb{N},\left\{n \in \mathbb{Z}^{+} \mid\{n, n+1, \cdots, n+k\} \subset F\right\}$ is syndetic;

(4) an IP set, if there is a subset $\left\{p_{i} \mid i \in \mathbb{N}\right\}$ such that $F \supset\left\{p_{i_{1}}+\cdots+p_{i_{k}} \mid k \in \mathbb{N}, i_{1}<\cdots<i_{k}\right\}$.

Denote the collection of all syndetic (resp., thick, thickly syndetic, IP) subsets of $\mathbb{Z}^{+}$by $\mathscr{F}_{\mathrm{s}}$ (resp., $\mathscr{F}_{\mathrm{t}}$, $\mathscr{F}_{\mathrm{ts}} \mathscr{F}_{\text {ip }}$. It can be verified that $\mathrm{\kappa} \mathscr{F}_{\mathrm{s}}=\mathscr{F}_{\mathrm{t}}$.

Let $\mathscr{F}$ be a Furstenberg family. A NADS is $\mathscr{F}$-transitive, if for any pair of nonempty open subsets $\mathrm{U}, \mathrm{V}$ of $\mathrm{X}, \mathrm{N}(\mathrm{U}, \mathrm{V}) \in \mathscr{F}$. A point $\mathrm{x} \in \mathrm{X}$ is an $\mathscr{F}$-recurrent point of $\left\{\mathrm{f}_{\mathfrak{n}}\right\}_{\mathfrak{n}=1}^{\infty}$, if for any neighborhood $\mathrm{U}$ of $x,\left\{n \in \mathbb{Z}^{+} \mid F_{1}^{n}(x) \in U\right\} \in \mathscr{F}$. A $\mathscr{F}_{\text {s }}$-recurrent point (resp., $\mathscr{F}_{\text {inf }}$-recurrent point) is called an almost periodic point (resp., recurrent point) of $\left\{f_{\mathfrak{n}}\right\}_{\mathfrak{n}=1}^{\infty}$. The set of all almost periodic points (resp., recurrent points) of $\left\{f_{n}\right\}_{\mathfrak{n}=1}^{\infty}$ is denoted by $\operatorname{AP}\left(\left\{f_{n}\right\}_{\mathfrak{n}=1}^{\infty}\right)$ (resp., $\operatorname{Rec}\left(\left\{f_{\mathfrak{n}}\right\}_{\mathfrak{n}=1}^{\infty}\right)$ ). A pair $(x, y) \in X \times X$ is proximal, if $\liminf _{n \rightarrow \infty} \rho\left(F_{1}^{n}(x), F_{1}^{n}(y)\right)=0$.

For $\mathrm{U} \subset \mathrm{X}$ and $\varepsilon>0$, let

$$
\mathrm{N}(\mathrm{U}, \varepsilon)=\left\{n \in \mathbb{Z}^{+} \mid \operatorname{diam}\left(\mathrm{F}_{1}^{\mathrm{n}}(\mathrm{U})\right) \geqslant \varepsilon\right\} .
$$

It is easy to see that a NADS $\left(X,\left\{f_{n}\right\}_{n=1}^{\infty}\right)$ is sensitive if and only if there exists $\varepsilon>0$ such that for any nonempty open subset $U \subset X, N(U, \varepsilon) \neq \varnothing$. For an ADS, Moothathu [10] initiated a preliminary study of stronger forms of sensitivity formulated in terms of some subsets of $\mathbb{Z}^{+}$, namely the syndetical sensitivity and cofinite sensitivity. Similarly to Moothathu [10], a NADS $\left(X,\left\{f_{n}\right\}_{n=1}^{\infty}\right)$ is said to be:

(1) syndetically sensitive, if there exists $\varepsilon>0$ such that for any nonempty open subset $U \subset X, N(U, \varepsilon)$ is syndetic;

(2) cofinitely sensitive, if there exists $\varepsilon>0$ such that for any nonempty open subset $U \subset X, N(U, \varepsilon)$ is cofinite;

(3) multi-sensitive, if there exists $\varepsilon>0$ (multi-sensitive constant) such that for any $k \in \mathbb{N}$ and nonempty open subsets $U_{1}, \cdots, U_{k} \subset X, \bigcap_{i=1}^{k}\left\{n \in \mathbb{Z}^{+} \mid \operatorname{diam}\left(F_{1}^{n}\left(U_{i}\right)\right)>\varepsilon\right\} \neq \varnothing$. 
(4) $\mathscr{F}$-sensitive ( $\mathscr{F}$-sensitive constant), if there exists $\varepsilon>0$ such that for any nonempty open subset $\mathrm{U} \subset \mathrm{X}, \mathrm{N}(\mathrm{U}, \varepsilon) \in \mathscr{F}$, where $\mathscr{F}$ is a Furstenberg family.

Banks et al. [2] proved that every transitive ADS whose periodic points are dense in the state space has sensitive dependence on initial conditions. Based on this result, Lan [8] posed the following open problem (Problem 1.1). Wu et al. [15] proved that an ADS with $\bar{d}$-shadowing or $\mathrm{d}$-shadowing and a dense set of minimal points is totally syndetically sensitive. Li et al. [9, 12] studied relations of various types of sensitivity between an ADS and its induced ADS on the space of probability measures. Then, Wu and Chen [14] discussed the sensitivity and transitivity of fuzzified dynamical systems. For more recent results on the notion of sensitivity, one is referred to [11, 13, 16, 17, 19, 20, 22] and references therein.

Problem 1.1 ([8, Problem 1]). In non-autonomous dynamical systems, does transitivity together with periodic density imply sensitivity?

$\mathrm{Wu}$ and $\mathrm{Zhu}$ [21] proved the following results for the uniform convergence of NADS.

Lemma 1.2 ([21, Corollary 2.2]). Assume that NADS $\left(X,\left\{f_{n}\right\}_{n=1}^{\infty}\right)$ converges uniformly to a map $f$. Then for any $\varepsilon>0$ and any $k \in \mathbb{N}$, there exist $\xi(\varepsilon)>0$ and $N(k) \in \mathbb{N}$ such that for any $x, y \in X$ with $\rho(x, y)<\xi$ and any $n \geqslant N, \rho\left(F_{n}^{k}(x), F_{n}^{k}(y)\right)<\varepsilon$.

In this paper, we firstly give a negative answer to Problem 1.1 and obtain a sufficient condition under which a NADS is syndetically sensitive. Then, we prove that a product system is multi-sensitive (resp., $\mathscr{F}$-sensitive) if and only if there exists a factor system is multi-sensitive (resp., $\mathscr{F}$-sensitive), where $\mathscr{F}$ is a filterdual.

\section{Sensitivity for NADS}

Firstly, we construct a transitive NADS with strongly periodic density to negatively answer Problem 1.1 (see Example 2.1).

Example 2.1. Fix an equicontinuous transitive homeomorphism $(X, f)$ such that $X$ is infinite. Clearly, such a dynamical system exists. Take a NADS $\left(X,\left\{f_{n}\right\}_{n=1}^{\infty}\right)$ as $f_{2 n}=f^{-n}$ and $f_{2 n-1}=f^{n}$ for all $n \in \mathbb{N}$. For any $x \in X$, the following statements hold:

(a) $\mathrm{F}_{1}^{2 \mathrm{n}}(\mathrm{x})=\mathrm{x}, \forall \mathrm{n} \geqslant 1$

(b) $\mathrm{F}_{1}^{2 n-1}(x)=\mathrm{f}^{\mathrm{n}}(\mathrm{x}), \forall \mathrm{n} \geqslant 1$.

These imply that $\left(X,\left\{f_{n}\right\}_{n=1}^{\infty}\right)$ is equicontinuous and $\operatorname{Per}\left(\left\{f_{n}\right\}_{n=1}^{\infty}\right)=\operatorname{SP}\left(\left\{f_{n}\right\}_{n=1}^{\infty}\right)=X$. For any nonempty open subsets $U, V$ of $X$, condition (b) together with the transitivity of $(X, f)$ implies that $\left(X,\left\{f_{\mathfrak{n}}\right\}_{\mathfrak{n}=1}^{\infty}\right)$ is transitive. This example shows that the answer to Problem 1.1 is negative.

Next example shows that the sensitivity of every $f_{n}$ can not ensure their uniform convergence map is sensitive.

Example 2.2. Let $X=[0,1]$ and define $f_{n}: X \longrightarrow X$ as

$$
f_{n}(x)=\left\{\begin{array}{cc}
\frac{1-|1-2 n x|}{n}, & x \in\left[0, \frac{1}{n}\right], \\
\vdots & \vdots \\
f_{n}\left(x-\frac{k}{n}\right), & x \in\left[\frac{k}{n}, \frac{k+1}{n}\right], \\
\vdots & \vdots \\
f_{n}\left(x-\frac{n-1}{n}\right), & x \in\left[\frac{n-1}{n}, 1\right] .
\end{array}\right.
$$

It can be verified that each $f_{n}$ is sensitive and $\left\{f_{n}\right\}_{\mathfrak{n}=1}^{\infty}$ converges uniformly to $f \equiv 0$. Clearly, $f$ is not sensitive. 
Theorem 2.3. Let $\left(X,\left\{f_{n}\right\}_{n=1}^{\infty}\right)$ be a transitive NADS which converges uniformly to a map $\mathrm{f}$. If there exists some point $z \in X$ such that $\overline{\left\{F_{i}^{n}(z) \mid i, n \in \mathbb{Z}^{+}\right\}} \varsubsetneqq X$ and $\overline{\operatorname{AP}\left(\left\{f_{n}\right\}_{n=1}^{\infty}\right)}=X$, then $\left(X,\left\{f_{n}\right\}_{n=1}^{\infty}\right)$ is syndetically sensitive.

Proof. Fix a point $y \in X \backslash \overline{\left\{F_{i}^{n}(z) \mid i, n \in \mathbb{Z}^{+}\right\}}$and put $\varepsilon=\frac{1}{4} \inf \left\{\rho(x, y) \mid x \in \overline{\left\{F_{i}^{n}(z) \mid i, n \in \mathbb{Z}^{+}\right\}}\right\}>0$, $V=\{x \in X \mid \rho(x, y)<\varepsilon\}$. For any nonempty open subset $U \subset X$, noting that $\overline{\operatorname{AP}\left(\left\{f_{n}\right\}_{n=1}^{\infty}\right)}=X$, it can be verified that $N(U, V)$ is syndetic, i.e., there exists $M_{1} \in \mathbb{N}$ such that for any $n \in \mathbb{Z}^{+}$,

$$
\left[n, n+M_{1}\right] \cap N(U, V) \neq \varnothing .
$$

Applying Lemma 1.2 yields that there exist $\delta>0$ and $N \in \mathbb{N}$ such that for any $x \in X$ with $\rho(x, z)<\delta$ and any $n \geqslant N, \rho\left(F_{n}^{k}(x), F_{n}^{k}(z)\right)<\varepsilon$ holds for all $k=0,1, \cdots, M_{1}$. Set $W=\{x \in X \mid \rho(x, z)<\delta\}$. Then, there exists $M_{2} \in \mathbb{N}$ such that for any $n \in \mathbb{Z}^{+},\left[n, n+M_{2}\right] \cap N(U, W) \neq \varnothing$. This implies that for any fixed $n \geqslant N$, there exist $j \in\left[0, M_{2}\right]$ and $u \in U$ such that $F_{1}^{n+j}(u) \in W$. Thus, for any $0 \leqslant k \leqslant M_{1}$,

$$
\rho\left(F_{n+j}^{k}\left(F_{1}^{n+j}(u)\right), F_{n+j}^{k}(z)\right)=\rho\left(F_{1}^{n+j+k}(u), F_{n+j}^{k}(z)\right)<\varepsilon .
$$

Combining this with the choice of $\varepsilon$, it follows that for any $x \in V$,

$$
\rho\left(F_{1}^{n+j+k}(u), x\right) \geqslant 2 \varepsilon .
$$

Applying (2.1) implies that there exist $0 \leqslant k_{1} \leqslant M_{1}$ and $u^{\prime} \in U$ such that $F_{1}^{n+j+k_{1}}\left(u^{\prime}\right) \in V$. This, together with (2.2) implies that

$$
\rho\left(F_{1}^{n+j+k_{1}}(u), F_{1}^{n+j+k_{1}}\left(u^{\prime}\right)\right) \geqslant 2 \varepsilon \text {, i.e., } n+j+k_{1} \in N(U, 2 \varepsilon) .
$$

Hence, $\left(X,\left\{f_{\mathfrak{n}}\right\}_{\mathfrak{n}=1}^{\infty}\right)$ is syndetically sensitive as $U$ is arbitrary.

Furstenberg [5] proved that the following result holds for ADS.

Proposition 2.4. Let $\left(\mathrm{X},\left\{\mathrm{f}_{\mathfrak{n}}\right\}_{\mathfrak{n}=1}^{\infty}\right)$ be a NADS which converges uniformly to a map $\mathrm{f}$ and $(\mathrm{x}, \mathrm{y}) \in \mathrm{X} \times \mathrm{X}$. If $(\mathrm{x}, \mathrm{y})$ is proximal, then for any $\varepsilon>0,\left\{n \in \mathbb{Z}^{+}: \rho\left(\mathrm{F}_{1}^{\mathfrak{n}}(\mathrm{x}), \mathrm{F}_{1}^{\mathrm{n}}(\mathrm{y})\right)<\varepsilon\right\} \in \mathscr{F}_{\mathrm{t}}$.

Proof. Given any fixed $\varepsilon>0$ and for any $k \in \mathbb{N}$, applying Lemma 1.2 implies that there exists $\xi>0$ and $N \in \mathbb{N}$ such that for any $x_{1}, y_{1} \in X$ with $\rho\left(x_{1}, y_{1}\right)<\xi$ and any $0 \leqslant i \leqslant k, \rho\left(F_{n}^{i}\left(x_{1}\right), F_{n}^{i}\left(y_{1}\right)\right)<\varepsilon$ holds for any $n \geqslant k$. Since $(x, y)$ is proximal, there exists $m>N$ such that $\rho\left(F_{1}^{m}(x), F_{1}^{m}(y)\right)<\xi$. This, together with the choice of $\xi$, implies that for any $0 \leqslant i \leqslant k$,

$$
\rho\left(F_{1}^{m+i}(x), F_{1}^{m+i}(y)\right)=\rho\left(F_{m}^{i}\left(F_{1}^{m}(x)\right), F_{m}^{i}\left(F_{1}^{m}(y)\right)\right)<\varepsilon .
$$

Therefore, $\left\{n \in \mathbb{Z}^{+} \mid \rho\left(F_{1}^{n}(x), F_{1}^{n}(y)\right)<\varepsilon\right\} \in \mathscr{F}_{\text {t }}$.

\section{Multi-sensitivity and $\mathscr{F}$-sensitivity for NADS}

Let $\left(X,\left\{f_{\mathfrak{n}}\right\}_{\mathfrak{n}=1}^{\infty}\right)$ and $\left(Y,\left\{g_{\mathfrak{n}}\right\}_{\mathfrak{n}=1}^{\infty}\right)$ be two NADS. The product metric $\rho$ on $X \times Y$ is defined by

$$
\rho\left(\left(x_{1}, y_{1}\right),\left(x_{2}, y_{2}\right)\right)=\sqrt{\rho_{1}^{2}\left(x_{1}, x_{2}\right)+\rho_{2}^{2}\left(y_{1}, y_{2}\right)}
$$

for any $\left(x_{1}, y_{1}\right),\left(x_{2}, y_{2}\right) \in X \times Y$. Define their product system as $\left(X \times Y,\left\{f_{\mathfrak{n}} \times g_{n}\right\}_{\mathfrak{n}=1}^{\infty}\right)$ and call $\left(X,\left\{f_{n}\right\}_{n=1}^{\infty}\right)$ and $\left(Y,\left\{g_{\mathfrak{n}}\right\}_{\mathfrak{n}=1}^{\infty}\right)$ factor system of $\left(X \times Y,\left\{f_{\mathfrak{n}} \times g_{\mathfrak{n}}\right\}_{\mathfrak{n}=1}^{\infty}\right)$.

Recently, Wu et al. [18] proved that $(X \times Y, f \times g)$ is multi-sensitive if and only if $(X, f)$ or $(Y, g)$ is multi-sensitive. 
Theorem 3.1. Let $\left(\mathrm{X},\left\{\mathrm{f}_{\mathrm{n}}\right\}_{\mathfrak{n}=1}^{\infty}\right)$ and $\left(\mathrm{Y},\left\{\mathrm{g}_{\mathfrak{n}}\right\}_{\mathfrak{n}=1}^{\infty}\right)$ be two NADS. Then, $\left(\mathrm{X} \times \mathrm{Y},\left\{\mathrm{f}_{\mathfrak{n}} \times \mathrm{g}_{\mathfrak{n}}\right\}_{\mathfrak{n}=1}^{\infty}\right)$ is multi-sensitive if and only if $\left(\mathrm{X},\left\{\mathrm{f}_{\mathfrak{n}}\right\}_{\mathfrak{n}=1}^{\infty}\right)$ or $\left(\mathrm{Y},\left\{\mathrm{g}_{\mathfrak{n}}\right\}_{\mathfrak{n}=1}^{\infty}\right)$ is multi-sensitive.

Proof.

$(\Longleftarrow)$ Assume that $\left(X,\left\{f_{\mathfrak{n}}\right\}_{\mathfrak{n}=1}^{\infty}\right)$ is multi-sensitive with a multi-sensitive constant $\varepsilon>0$. For any

$k \in \mathbb{N}$ and any nonempty open subsets $W_{1}, \cdots, W_{k}$ of $X \times Y$, their exists nonempty open subsets $U_{1}, \cdots, U_{k}$ of $X$ and $V_{1}, \cdots, V_{k}$ of $Y$ such that $U_{i} \times V_{i} \subset W_{i}$ for any $1 \leqslant i \leqslant k$. Since $\left\{f_{n}\right\}_{n=1}^{\infty}$ is multi-sensitive, there exists $n \in \mathbb{N}$ such that for any $1 \leqslant i \leqslant k$, $\operatorname{diam}\left(F_{1}^{n}\left(U_{i}\right)\right) \geqslant \varepsilon$, implying that

$$
\operatorname{diam}\left(\mathrm{F}_{1}^{\mathrm{n}} \times \mathrm{G}_{1}^{\mathfrak{n}}\left(\mathrm{W}_{\mathfrak{i}}\right)\right) \geqslant \operatorname{diam}\left(\mathrm{F}_{1}^{\mathrm{n}} \times \mathrm{G}_{1}^{\mathfrak{n}}\left(\mathrm{U}_{\mathrm{i}} \times \mathrm{V}_{\mathfrak{i}}\right)\right) \geqslant \varepsilon,
$$

where $G_{1}^{n}=g_{n} \circ \cdots \circ g_{1}$, i.e., $\left(X \times Y,\left\{f_{n} \times g_{n}\right\}_{n=1}^{\infty}\right)$ is multi-sensitive.

$(\Longrightarrow)$ Let $\varepsilon>0$ be a multi-sensitive constant of $\left\{f_{\mathfrak{n}} \times g_{\mathfrak{n}}\right\}_{\mathfrak{n}=1}^{\infty}$ and suppose that both $\left\{f_{\mathfrak{n}}\right\}_{\mathfrak{n}=1}^{\infty}$ and $\left\{g_{\mathfrak{n}}\right\}_{\mathfrak{n}=1}^{\infty}$ are not multi-sensitive. Then there exist $k_{1}, k_{2} \in \mathbb{N}$, and nonempty open subsets $U_{1}, \cdots, U_{k_{1}}$ of $X$, $V_{1}, \cdots, V_{k_{2}}$ of $Y$, such that for any $n \in \mathbb{Z}^{+}$, there exist $i_{n} \in\left\{1, \cdots, k_{1}\right\}$ and $j_{n} \in\left\{1, \cdots, k_{2}\right\}$ satisfying

$$
\operatorname{diam}\left(\mathrm{F}_{1}^{\mathrm{n}}\left(\mathrm{U}_{\mathrm{i}_{\mathfrak{n}}}\right)\right) \leqslant \frac{\varepsilon}{2 \sqrt{2}} \text { and } \operatorname{diam}\left(\mathrm{G}_{1}^{\mathrm{n}}\left(\mathrm{V}_{\mathbf{j}_{\mathfrak{n}}}\right)\right) \leqslant \frac{\varepsilon}{2 \sqrt{2}} .
$$

Take $W_{i, j}=U_{i} \times V_{j}\left(1 \leqslant i \leqslant k_{1}, 1 \leqslant j \leqslant k_{2}\right)$. Clearly, each $W_{i, j}$ is a nonempty open subset of $X \times Y$. Since $\left\{f_{n} \times g_{n}\right\}_{n=1}^{\infty}$ is multi-sensitive, there exists $m \in \mathbb{Z}^{+}$such that for any $i \in\left\{1, \cdots, k_{1}\right\}$ and any $j \in\left\{1, \cdots, k_{2}\right\}$,

$$
\operatorname{diam}\left(F_{1}^{m} \times G_{1}^{m}\left(W_{i, j}\right)\right)=\operatorname{diam}\left(F_{1}^{m}\left(U_{i}\right) \times G_{1}^{m}\left(V_{j}\right)\right) \geqslant \varepsilon .
$$

This, together with (3.1), implies that

$$
\frac{\varepsilon}{2}=\sqrt{\left(\frac{\varepsilon}{2 \sqrt{2}}\right)^{2}+\left(\frac{\varepsilon}{2 \sqrt{2}}\right)^{2}} \geqslant \operatorname{diam}\left(\mathrm{F}_{1}^{\mathrm{m}}\left(\mathrm{U}_{\mathrm{i}}\right) \times \mathrm{G}_{1}^{\mathrm{m}}\left(\mathrm{V}_{\mathrm{j}}\right)\right) \geqslant \varepsilon,
$$

which is a contradiction.

Corollary 3.2. Let $\left(X,\left\{f_{n}\right\}_{n=1}^{\infty}\right)$ and $\left(Y,\left\{g_{n}\right\}_{n=1}^{\infty}\right)$ be two NADS. Then, $\left(X \times Y,\left\{f_{n} \times g_{n}\right\}_{\mathfrak{n}=1}^{\infty}\right)$ is sensitive if and only if $\left(\mathrm{X},\left\{\mathrm{f}_{\mathrm{n}}\right\}_{\mathfrak{n}=1}^{\infty}\right)$ or $\left(\mathrm{Y},\left\{\mathrm{g}_{\mathrm{n}}\right\}_{\mathfrak{n}=1}^{\infty}\right)$ is sensitive.

Theorem 3.3. Let $\left(X,\left\{\mathrm{f}_{\mathrm{n}}\right\}_{\mathfrak{n}=1}^{\infty}\right)$ and $\left(\mathrm{Y},\left\{\mathrm{g}_{\mathrm{n}}\right\}_{\mathrm{n}=1}^{\infty}\right)$ be two NADS and $\mathscr{F}$ be a filterdual. Then,

$$
\left(X \times Y,\left\{f_{\mathfrak{n}} \times g_{n}\right\}_{\mathfrak{n}=1}^{\infty}\right),
$$

is $\mathscr{F}$-sensitive if and only if $\left(\mathrm{X},\left\{\mathrm{f}_{\mathfrak{n}}\right\}_{\mathfrak{n}=1}^{\infty}\right)$ or $\left(\mathrm{Y},\left\{\mathrm{g}_{\mathfrak{n}}\right\}_{\mathfrak{n}=1}^{\infty}\right)$ is $\mathscr{F}$-sensitive.

Proof.

$(\Longleftarrow)$ Similarly to the proof of Theorem 3.1, this holds trivially.

$(\Longrightarrow)$ Suppose that $\left\{f_{\mathfrak{n}} \times g_{\mathfrak{n}}\right\}_{\mathfrak{n}=1}^{\infty}$ is $\mathscr{F}$-sensitive with an $\mathscr{F}$-sensitive constant $\varepsilon>0$ and that both $\left\{f_{\mathfrak{n}}\right\}_{\mathfrak{n}=1}^{\infty}$ and $\left\{g_{n}\right\}_{n=1}^{\infty}$ are not $\mathscr{F}$-sensitive. Then there exist nonempty open subsets $U$ of $X, V$ of $Y$ such that $\mathrm{N}(\mathrm{U}, \varepsilon / 2 \sqrt{2}) \notin \mathscr{F}$ and $\mathrm{N}(\mathrm{V}, \varepsilon / 2 \sqrt{2}) \notin \mathscr{F}$, implying that

$$
\mathrm{F}_{1}:=\mathbb{Z}^{+} \backslash \mathrm{N}(\mathrm{U}, \varepsilon / 2 \sqrt{2})=\left\{\mathrm{n} \in \mathbb{Z}^{+} \mid \operatorname{diam}\left(\mathrm{F}_{1}^{\mathrm{n}}(\mathrm{U})\right) \geqslant \varepsilon / 2 \sqrt{2}\right\} \in \kappa \mathscr{F},
$$

and

$$
\mathrm{F}_{2}:=\mathbb{Z}^{+} \backslash \mathrm{N}(\mathrm{V}, \varepsilon / 2 \sqrt{2})=\left\{\mathrm{n} \in \mathbb{Z}^{+} \mid \operatorname{diam}\left(\mathrm{G}_{1}^{\mathrm{n}}(\mathrm{V})\right) \geqslant \varepsilon / 2 \sqrt{2}\right\} \in \kappa \mathscr{F} .
$$

As $\mathscr{F}$ is a filterdual, then $\mathrm{F}=\mathrm{F}_{1} \cap \mathrm{F}_{2} \in \mathrm{\kappa} \mathscr{F}$. Take a nonempty open subset $\mathrm{W}=\mathrm{U} \times \mathrm{V} \subset \mathrm{X} \times \mathrm{Y}$. It can be verified that for any $n \in F$,

$$
\operatorname{diam}\left(F_{1}^{n} \times G_{1}^{n}(W)\right) \leqslant \sqrt{\operatorname{diam}^{2}\left(F_{1}^{n}(U)\right)+\operatorname{diam}^{2}\left(G_{1}^{n}(V)\right)} \leqslant \frac{\varepsilon}{2} .
$$

This implies that

$$
\mathscr{F} \ni \mathrm{N}(\mathrm{U} \times \mathrm{V}, \varepsilon) \subset \mathbb{Z}^{+} \backslash \mathrm{F} \notin \mathscr{F},
$$

which is a contradiction as $\mathscr{F}$ is hereditary upwards. 


\section{Acknowledgment}

This project was supported by the National Natural Science Foundation of China (No. 11601449, 11401495, 11501391), the Opening Project of Artificial Intelligence Key Laboratory of Sichuan Province (No. 2015RZJ01) and the scientific research starting project of Southwest Petroleum University (No. 2015QHZ029).

\section{References}

[1] E. Akin, Recurrence in topological dynamics, Furstenberg families and Ellis actions, The University Series in Mathematics, Plenum Press, New York, (1997). 1

[2] J. Banks, J. Brooks, G. Cairns, G. Davis, P. Stacey, On Devaney's definition of chaos, Amer. Math. Monthly, 99 (1992), 332-334. 1

[3] S. N. Elaydi, Nonautonomous difference equations: open problems and conjectures, Differences and differential equations, Fields Inst. Commun., Amer. Math. Soc., Providence, RI, 42 (2004), 423-428. 1

[4] S. Elaydi, R. J. Sacker, Nonautonomous Beverton-Holt equations and the Cushing-Henson conjectures, J. Difference Equ. Appl., 11 (2005), 337-346. 1

[5] H. Furstenberg, Recurrence in ergodic theory and combinatorial number theory, M. B. Porter Lectures, Princeton University Press, Princeton, N.J., (1981). 2

[6] S. Kolyada, M. Misiurewicz, L. Snoha, Topological entropy of nonautonomous piecewise monotone dynamical systems on the interval, Fund. Math., 160 (1999), 161-181. 1

[7] S. Kolyada, L. Snoha, Topological entropy of nonautonomous dynamical systems, Random Comput. Dynam., 4 (1996), 205-233. 1

[8] Y.-Y. Lan, Chaos in nonautonomous discrete fuzzy dynamical systems, J. Nonlinear Sci. Appl., 9 (2016), 404-412. 1, 1, 1.1

[9] J. Li, P. Oprocha, X.-X. Wu, Furstenberg families, sensitivity and the space of probability measures, Nonlinearity, 30 (2017), 987-1005. 1

[10] T. K. S. Moothathu, Stronger forms of sensitivity for dynamical systems, Nonlinearity, 20 (2007), 2115-2126. 1

[11] Y.-G. Wang, G. Wei, W. H. Campbell, Sensitive dependence on initial conditions between dynamical systems and their induced hyperspace dynamical systems, Topology Appl., 156 (2009), 803-811. 1

[12] X.-X. Wu, Chaos of transformations induced onto the space of probability measures, Internat. J. Bifur. Chaos Appl. Sci. Engrg., 26 (2016), 12 pages. 1

[13] X.-X. Wu, A remark on topological sequence entropy, Internat. J. Bifur. Chaos Appl. Sci. Engrg., 27 (2017), 7 pages. 1

[14] X.-X. Wu, G.-R. Chen, Sensitivity and transitivity of fuzzified dynamical systems, Inform. Sci., 396 (2017), 14-23. 1

[15] X.-X. Wu, P. Oprocha, G.-R. Chen, On various definitions of shadowing with average error in tracing, Nonlinearity, 29 (2016), 1942-1972. 1

[16] X.-X. Wu, X. Wang, On the iteration properties of large deviations theorem, Internat. J. Bifur. Chaos Appl. Sci. Engrg., 26 (2016), 6 pages. 1

[17] X.-X. Wu, X. Wang, Topological dynamics of Zadeh's extension on the space of upper semi-continuous fuzzy sets, ArXiv, 2016 (2016), 14 pages. 1

[18] X.-X. Wu, J.-J. Wang, G.-R. Chen, F-sensitivity and multi-sensitivity of hyperspatial dynamical systems, J. Math. Anal. Appl., 429 (2015), 16-26. 3

[19] X.-X. Wu, X. Wang, G.-R. Chen, On the large deviations theorem of weaker types, Internat. J. Bifur. Chaos Appl. Sci. Engrg., 27 (2017), 12 pages. 1

[20] X.-X. Wu, L.-D. Wang, G.-R. Chen, Weighted backward shift operators with invariant distributionally scrambled subsets, Ann. Funct. Anal., 8 (2017), 199-210. 1

[21] X.-X. Wu, P.-Y. Zhu, Chaos in a class of non-autonomous discrete systems, Appl. Math. Lett., 26 (2013), 431-436. 1, 1.2

[22] X.-D. Ye, R.-F. Zhang, On sensitive sets in topological dynamics, Nonlinearity, 21 (2008), 1601-1620. 1 